\title{
Microbiology of the conjunctival sac before and after cataract surgery
}

\author{
Patrycja Kuklo๑ ${ }^{1}$, Andrzej Grzybowski $\oplus^{2}$ \\ 'Department of Ophthalmology, University of Warmia and Mazury, Olsztyn, Poland \\ ${ }^{2}$ Institute for Research in Ophthalmology, Poznan, Poland
}

\begin{abstract}
BACKGROUND: The aim of the study was to evaluate the bacterial flora and antibiotic susceptibility profile of bacterial isolates taken from the conjunctival sacs of patients undergoing phacoemulsification cataract surgery, and to compare them.

MATERIAL AND METHODS: In total, 200 conjunctival swabs were collected from 50 patients between June and December of 2017.

RESULTS: The most common pathogen collected from the conjunctival sacs before surgery was coagulase-negative staphylococci (CoNS) (65\% of swabs); 45 of them (45\%) were methicillin-sensitive staphylococci (MS-CoNS) and 25 (25\%) were methicillin-resistant staphylococcus (MR-CoNS). Following the surgeries, CoNS were collected from 34 swabs (34\%), 16\% of which were taken from the eye on which the operation had been performed. Twenty-three swabs (23\%) were MS-CoNS and 11 (11\%) were MR-CoNS. The number of CoNS-positive swabs after pharmacotherapy decreased by $52 \%$. The possibility of obtaining sterile swabs was statistically and significantly higher in eyes in which chemoprophylaxis was used [(OR $=4.58,95 \% \mathrm{CI}: 2.91-7.21), \mathrm{p}<0.001)]$. The possibility of obtaining sterile swabs was not correlated with gender $(p=0.866)$ or diabetes $(p=0.712)$, but was observed more frequently in younger patients $(\mathrm{p}=0.001)$. Multi-drug resistant bacteria were detected in 34 swabs before surgery (34\%) and in 26 samples after operations (26\%).

CONCLUSION: It is probably impossible to sterilise the conjunctival sac. There is a risk of multi-drug resistant bacterial flora colonising the conjunctival sac.
\end{abstract}

KEY WORDS: conjunctival sac; cataract surgery; antibiotic susceptibility profile

Ophthalmol J 2020; Vol. 5, 71-75

\section{INTRODUCTION}

Endophthalmitis is rare, but it is one of the most devastating complications following cataract surgery, with a recorded frequency of $0.087 \%$ in 1990 and $0.265 \%$ after 2000 [1]. It can have heterogeneous etiologies and variable clinical manifestations, with a high risk of blindness, irreversible changes in the anatomy of the eye or even the loss of the eyeball. It is known that post-cataract endophthalmitis is caused by bacteria being transferred into the eyeball from the conjunctival sac, the eyelids, the eyelashes or the periorbital skin [2]. All these areas are colonised physiologically by bacteria. During operation, they are transferred to the normally sterile anterior chamber, which can be a risk factor for endophthalmitis [3]. The aim of this study was to describe the microbiological profile of the conjunctival sac before and after cataract surgery and to assess how perioperative prophylaxis affects changes in the bacterial flora.

\section{MATERIAL AND METHODS}

From June to December 2017, 200 conjunctival swabs were collected from 50 patients undergoing 
phacoemulsification cataract surgery. Four swabs were obtained from each patient. The first two swabs from each eye were collected on admission to the hospital before any eye-drops were administered. The second two samples were taken from each eye 20 minutes after the cataract surgery. Each patient received either four drops of topical $0.3 \%$ ofloxacin every 15 minutes one hour before surgery in the eye to be operated upon, or two drops of antibiotic on the day of admission and another two drops the next day, 30 and 15 minutes before surgery, respectively. Concomitant medications to the operated eye contained one drop of $1 \%$ tropicamide, one drop of $10 \%$ phenylephrine and one drop of diclofenac. Routine prophylaxis in the operating theatre included a $5 \%$ povidone-iodine solution for surface disinfection of the eyelids and skin around the orbit, $5 \%$ povidone-iodine for three minutes administered to the conjunctival sac before operation, and intracameral injection of $1 \mathrm{mg} 0.1 \mathrm{~mL}$ cefuroxime at the end of the procedure. On the day of admission, one drop of $1 \%$ tropicamide was administered to the eye that was not to be operated upon as part of the routine examination. The exclusion criteria included topical and systemic antibiotic therapy one month prior to the surgery, glaucoma that was being treated with eye-drops, keratoconjunctivitis sicca, and orbital or ocular inflammation one month prior to the surgery. The same examiner collected all the samples in this study, thus ensuring that the collecting procedure was consistent for all the samples taken. All the tests were analysed in the same microbiological laboratory. All the patients signed an informed consent form approved by the local ethics committee.

\section{RESULTS}

Forty-nine patients were included in the study. Thirty one were female $(61.0 \%)$ and 19 were male $(38.0 \%)$. The mean age of the patients was 71.81 ( $\mathrm{SD}=8.86$ years). The female group was statistically significantly older than was the male group. The mean age of the female group was $73.91(\mathrm{SD}=7.69)$, while it was $68.05( \pm 9.76)$ for the male group $(p=0.031)$. Thirty per cent of the patients were diabetic. Twenty-seven patients $(19 \mathrm{fe}-$ males and eight males) operation to the left eye, and 22 patients ( 12 females and 10 males) to the right eye, $(p=0.389)$. Two hundred conjunctival swabs were collected. Sterile samples were collected from 14 eyes $(14 \%)$ prior to the operations. Fol-

\begin{tabular}{|c|c|}
\hline Trait & Statistics \\
\hline \multicolumn{2}{|l|}{ Sex } \\
\hline Females, n (\%) & $31(62.0)$ \\
\hline Males, n (\%) & $19(38,0)$ \\
\hline \multicolumn{2}{|l|}{ Age (years) } \\
\hline Overall, mean (SD) & $71.81(8.86)$ \\
\hline Females, mean (SD) & $73.91(7.69)$ \\
\hline Males, mean (SD) & $68.05(9.76)$ \\
\hline Diabetes mellitus, $\mathbf{n}(\%)$ & $15(30.0)$ \\
\hline \multicolumn{2}{|l|}{ Eye operated } \\
\hline Left, n (\%) & $28(56.0)$ \\
\hline Right, n (\%) & $22(44.0)$ \\
\hline
\end{tabular}

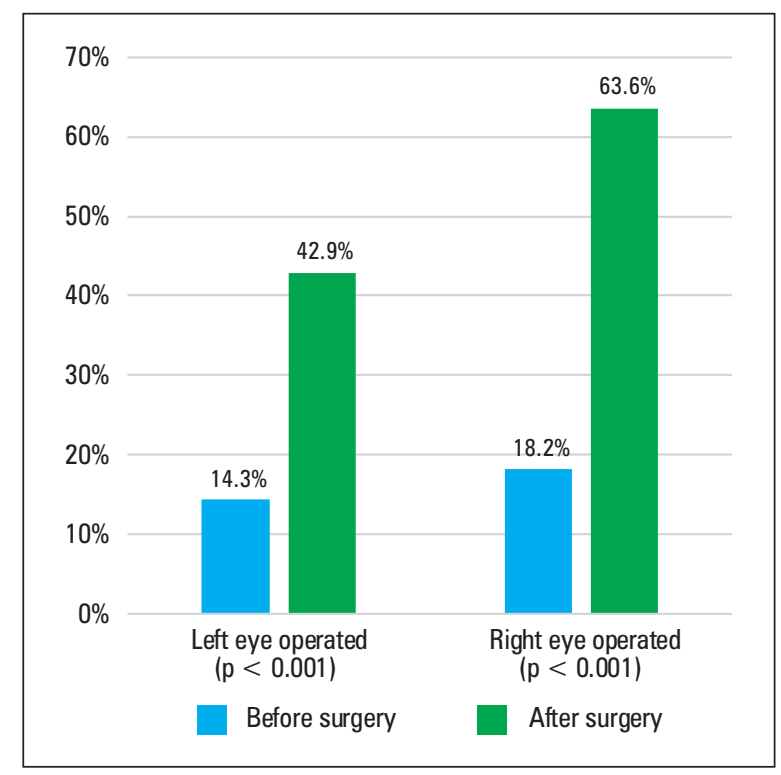

FIGURE 1. Sterility of conjunctival sac before and after cataract surgery in the examined patient who had undergone chemoprophylaxis by operated eye $(p<0.001)$

lowing the surgeries, sterile samples were obtained from $26(52.0 \%)$ that had been operated upon and from $20(40 \%)$ that had not. The possibility of obtaining sterile swabs was statistically significantly higher for eyes in which chemoprophylaxis had been used $(\mathrm{OR}=4.58,95 \% \mathrm{CI}: 2.91-7.21$; $\mathrm{p}<0.001)$. A multifactor analysis with repeatable measurements showed that the possibility of obtaining sterile swabs was not correlated with gen$\operatorname{der}(\mathrm{p}=0.866)$ or diabetes $(\mathrm{p}=0.712)$, but was observed statistically more frequently in younger patients $(p=0.001)$. No patient developed endophthalmitis after surgery (Tab. 1, Fig. 1). 


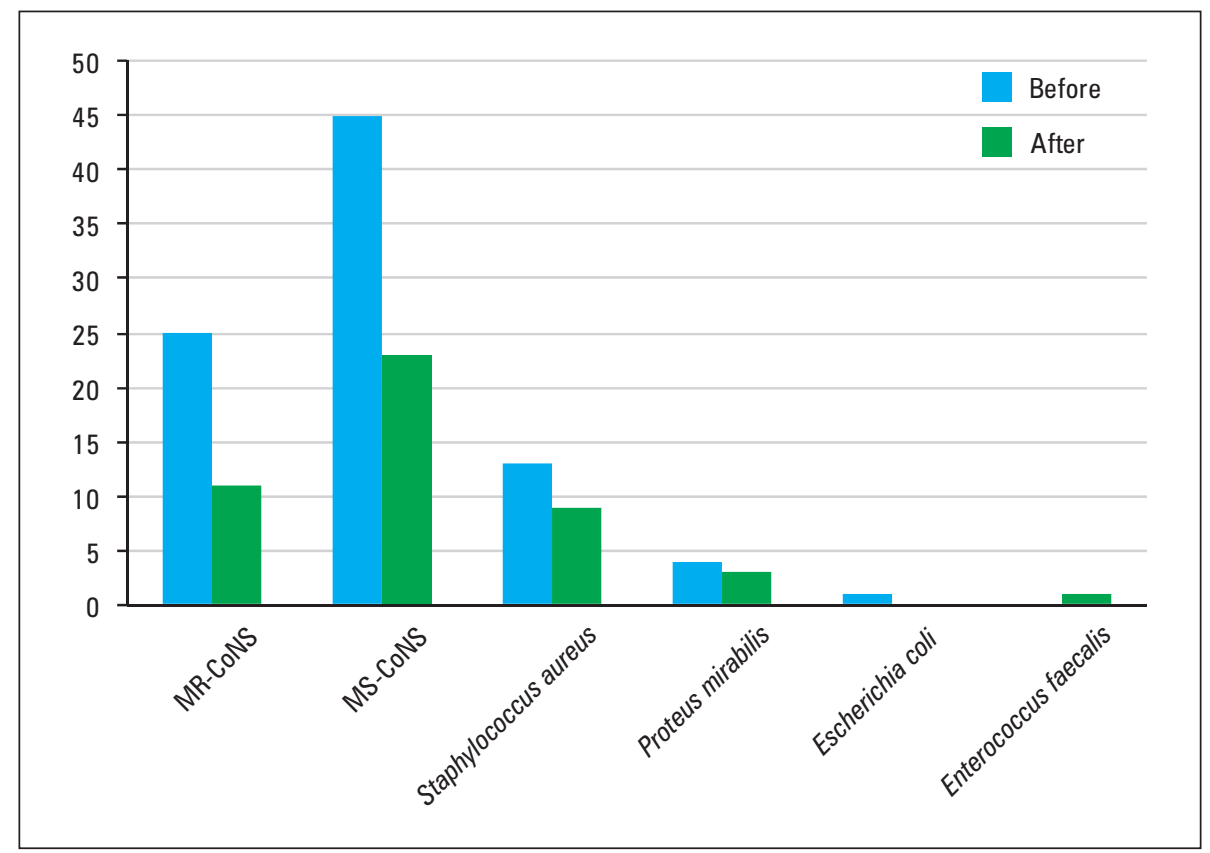

FIGURE 2. Percentage of bacteria cultured from the operated eye before and after cataract surgery. MR-CoNS — methicillin-resistant coagulase negative Staphylococci; MS-CoNS — methicillin-sensitive coagulase negative Staphylococci

Prior to the operations, CoNS were cultured from 65 samples (65\% of the swabs collected before the surgeries). Of them, 45 (45\%) were methicillin-sensitive (MS-CoNS) and 25 (25\%) were methicillin-resistant Staphylococci (MR-CoNS). After the surgeries, CoNS were obtained from 16 eyes that had been operated upon (16\%) and from 18 (18\%) that had not. Twenty-three (23\%) were MS-CoNS and $11(11 \%)$ were MR-CoNS. The number of CoNS-positive swabs after pharmacotherapy decreased by $52 \%$. Staphylococcus aureus was cultured from $13(13 \%)$ swabs obtained prior to the operations (five from the eyes that would be operated upon and eight from the eyes that would not) and in nine (9\%) samples following the surgeries. Three $(3 \%)$ positive samples were obtained from eyes that had received operations. Other pathogens cultivated before the surgeries included Proteus mirabilis from four (4\%) swabs and Escherichia coli from one swab (1\%). After the operations, Proteus mirabilis was cultured from three (3\%) samples and Enterococcus faecalis from one $(1 \%)$ sample taken from the eyes that had been operated upon. Proteus mirabilis was cultured from one (1\%) swab, Klebsiella spp. from one (1\%) swab, and Enterobacter cloacae from one (1\%) swab taken from the eyes that had not been operated upon. The patient with Klebsiella spp. was hospitalised for one day prior to the surgery, and the patient with Enterobacter cloacae was operated upon on the day of admission. Multi-drug resistant bacterial flora was detected in $34(34 \%)$ of the swabs taken before the surgeries, and in $26(26 \%)$ of the swabs taken following the operations from both the eyes that had been operated upon and those that had not. Multi-drug resistant CoNS were detected in $18(18 \%)$ swabs. Amongst the diabetic patients, the bacterial flora contained CoNS in 23 swabs (47\% of the samples from the diabetic patients versus 53\% from the non-diabetic patients), Staphylococcus aureus in eight swabs (16\% versus $8 \%)$ and Escherichia coli in one swab taken from a diabetic patient (Fig.2).

Sensitivity testing was performed for all the swabs. Prior to the surgeries, bacteria sensitive to all antibiotics were collected from 35 swabs (35\% samples taken before the operations); resistance to erythromycin was detected in 28 (28\%) swabs, to cloxacillin in $21(21 \%)$, to tobramycin in $19(19 \%)$, to gentamicin in $12(12 \%)$, to tetracycline in $12(12 \%)$, to ciprofloxacin in seven $(7 \%)$, to levofloxacin in six $(6 \%)$, to moxifloxacin in four $(4 \%)$, and to ofloxacin in seven $(7 \%)$ swabs. After the operations, bacteria sensitive to all antibiotics were collected from 23 eyes (23\%). Resistance to tobramycin was found in 20 swabs $(20 \%)$, to erythromycin in $16(16 \%)$, to cloxacylin in $15(15 \%)$, to gentamicin in $13(13 \%)$, to ciprofloxacin in nine $(9 \%)$, to tetracycline in 


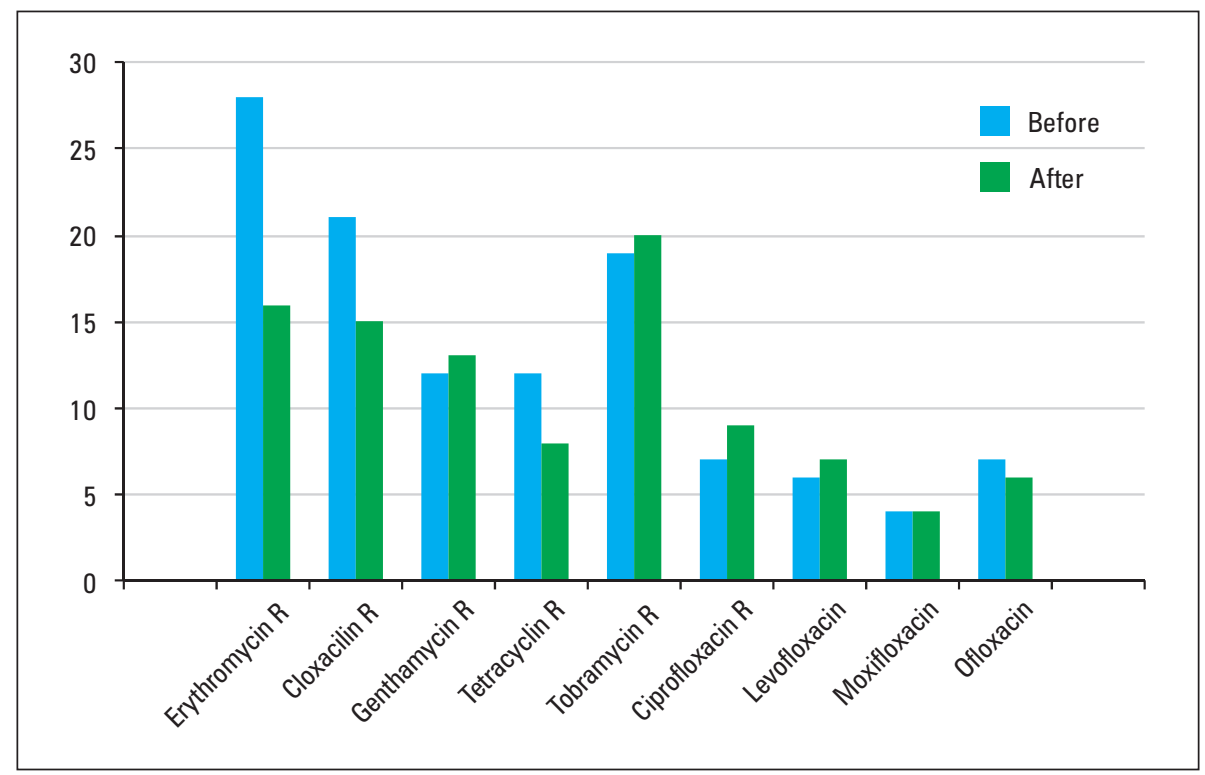

FIGURE 3. Bacteria susceptibility before and after the operation

eight $(8 \%)$, to levofloxacin in seven (7\%), to amikacin in six $(6 \%)$, to ofloxacin in six $(6 \%)$, and to moxifloxacin in four $(4 \%)$ swabs. The number of bacteria resistant to gentamicin, tobramycin, ciprofloxacin and levofloxacin increased after the operations $(12 \%$ vs. $13 \%, 19 \%$ vs. $20 \%, 7 \%$ vs. $9 \%$, and $6 \%$ vs. $7 \%$, respectively). The number of bacteria resistant to moxifloxacin was the same before and after the operations. A lower number of pathogens resistant to erythromycin, cloxacilline and tetracycline was observed. Multi-drug resistant bacteria were detected in 34 (34\%) swabs prior to the surgeries and in $26(26 \%)$ of the samples after the operations (Fig. 3).

\section{DISCUSSION}

In our study, the possibility of obtaining sterile swabs was statistically significantly higher from eyes in which chemoprophylaxis had been used. The possibility of obtaining sterile swabs was not linked to gender or diabetes, but was observed more frequently in younger patients. The most common bacterium collected from the conjunctival sacs before and after the operations was CoNS $(65 \%$ before the operation versus $34 \%$ after the surgery). The other pathogens were Staphylococcus aureus, Proteus mirabilis, Escherichia coli, Enterococcus faecalis, Klebsiella spp., and Enterobacter cloacae. The profiles of the collected microorganisms changed after the operations, which also led to changes in the antibiograms, with higher antibiotic resistance following the operations. Multi-drug resistant bacteria were detected in $34(34 \%)$ swabs before surgery and in $26(26 \%)$ samples after operation. Gram-positive CoNS (particularly Staphylococcus epidermidis) were the most common bacterial flora isolated from eyes with post-cataract endophthalmitis [4, 5]. The EVS study showed that the percentage of Gram-positive coagulase-negative bacteria isolated from postoperative endophthalmitis was $46.9 \%$ [6]. In our study, the most frequently isolated microorganism taken from the conjunctival sac before and after cataract surgery was coagulase-negative staphylococcus. This type of bacteria occurs naturally in the conjunctival sac and on the surrounding skin and lashes [5] and does not cause infection in these areas. Other Gram-positive microorganisms isolated from the conjunctiva constituted 15\% [6]. In our study, they were collected from $13(13 \%)$ swabs prior to the operations and from nine (9\%) swabs after the surgeries. The frequency of isolated microorganisms in our analysis is comparable to the frequency of pathogens isolated from eyes after endophthalmitis [6]. Hori et al. [7] noted the appearance of multi-drug resistant CoNS in conjunctival swabs taken prior to surgery. In our study, these pathogens were found in a high percentage of swabs taken both before $34 \%$ samples) and after surgery (26\%). This is potentially a serious problem because most of the antibiotics used in ophthalmology are useless in the treatment of infections based on this aetiology. Moreover, perioperative antibiotherapy is a potential risk factor for increased antibiotic resistance [8]. 
A correlation between diabetes mellitus and a greater risk of positive conjunctival swabs was confirmed in previous studies $[9,10]$. An increased prevalence of specific conjunctival bacterial flora, such as Staphylococcus aureus, Enterococci and Klebsiella spp., was also observed in diabetic patients. A greater incidence of positive cultured swabs was not confirmed in our study; however, the diabetic patients' bacterial flora contained CoNS in 23 swabs (47\% of the samples taken from diabetic patients), Staphylococcus aureus in swabs (16\%) and Escherichia coli in one swab. The ages and genders of the patients were identified as risk factors contributing to the cultured swabs that were positive in the previous studies [11, 12], but only younger patients had a lower risk of positive swabs taken from the conjunctiva in our study.

The intracameral concentration of topical preoperative antibiotics is too low to decrease the number of the most common microorganisms [13], and the ability of the most common conjunctival bacteria to form a biofilm [14] can reduce the effectiveness of perioperative antibiotherapy significantly.

In our study, sterile swabs were collected from 19\% of the eyes before and $47 \%$ of the eyes after the operations. This does not necessarily imply sterility in those eyes. No treatment was administered and the patients did not receive any eye-drops in 19\% of the sterile swabs that were collected before the operations. Considering the types of bacteria, some of the positive swabs that were taken after the operations could have been contaminated by the surrounding skin during the collection of the samples and could have been the result of the patient having poor hygiene.

Although a limitation of this study was the small sample size, the sample size is comparable to sample sizes in previous publications [7, 9-12].

\section{CONCLUSION}

Perioperative chemotherapy changes the profile of conjunctival bacteria and their susceptibility to antibiotics. Chemoprophylaxis increases the possibility of obtaining sterile swabs after an operation, but it also changes the profile of the bacteria in the conjunctival sac. It is probably impossible to sterilise the conjunctival sac. There is a risk that the conjunctival sac will be colonised by multi-drug resistant bacterial flora.

\section{Conflict of interests}

The authors declare that there is no conflict of interest.

\section{REFERENCES}

1. Taban M, Behrens A, Newcomb RL, et al. Acute endophthalmitis following cataract surgery: a systematic review of the literature. Arch Ophthalmol. 2005; 123(5): 613-620, doi: 10.1001/archopht.123.5.613, indexed in Pubmed: 15883279.

2. Bannerman $\mathrm{TL}$, Rhoden $\mathrm{DL}, \mathrm{McAllister} S \mathrm{SK}$, et al. The source of coagulase-negative staphylococci in the Endophthalmitis Vitrectomy Study. A comparison of eyelid and intraocular isolates using pulsed-field gel electrophoresis. Arch Ophthalmol. 1997; 115(3): 357-361, doi: 10.1001/archopht.1997.01100150359008, indexed in Pubmed: 9076208.

3. Leong J, Shah R, McCluskey P, et al. Bacterial contamination of the anterior chamber during phacoemulsification cataract surgery. $J$ Cataract Refract Surg. 2002; 28(5): 826-833, doi: 10.1016/s08863350(01)01160-9, indexed in Pubmed: 11978463.

4. Keynan Y, Finkelman Y, Lagacé-Wiens P. The microbiology of endophthalmitis: global trends and a local perspective. Eur J Clin Microbiol Infect Dis. 2012; 31(11): 2879-2886, doi: 10.1007/s10096-0121659-x, indexed in Pubmed: 22664925.

5. HAN D, WISNIEWSKI S, WILSON L, et al. Spectrum and Susceptibilities of Microbiologic Isolates in the Endophthalmitis Vitrectomy Study. Am J Ophthalmol. 1996; 122(1): 1-17, doi: 10.1016/s00029394(14)71959-2, indexed in Pubmed: 8659579.

6. Results of the Endophthalmitis Vitrectomy Study. Arch Ophthalmol. 1995; 113(12): 1479, doi: 10.1001/archopht.1995.01100120009001, indexed in Pubmed: 7487614.

7. Hori Y, Nakazawa T, Maeda N, et al. Susceptibility comparisons of normal preoperative conjunctival bacteria to fluoroquinolones. J Cataract Refract Surg. 2009; 35(3): 475-479, doi: 10.1016/j.jcrs.2008.11.049, indexed in Pubmed: 19251140.

8. Simunovic MP, Rush RB, Hunyor AP, et al. Endophthalmitis following intravitreal injection versus endophthalmitis following cataract surgery: clinical features, causative organisms and post-treatment outcomes. Br J Ophthalmol. 2012; 96(6): 862-866, doi: 10.1136/ bjophthalmol-2011-301439, indexed in Pubmed: 22446145.

9. Fernández-Rubio ME, Rebolledo-Lara L, Martinez-García M, et al. The conjunctival bacterial pattern of diabetics undergoing cataract surgery. Eye (Lond). 2010; 24(5): 825-834, doi: 10.1038/eye.2009.218, indexed in Pubmed: 19713978.

10. Hoshi S, Hashida M, Urabe K. Risk factors for aerobic bacterial conjunctival flora in preoperative cataract patients. Eye (Lond). 2016; 30(11): 1439-1446, doi: 10.1038/eye.2016.143, indexed in Pubmed: 27419840.

11. Rubio EF. Climatic influence on conjunctival bacteria of patients undergoing cataract surgery. Eye (Lond). 2004; 18(8): 778-784, doi: 10.1038/sj.eye.6701352, indexed in Pubmed: 15002018.

12. Hoshi S, Hashida M, Urabe K. Risk factors for aerobic bacterial conjunctival flora in preoperative cataract patients. Eye (Lond). 2016; 30(11): 1439-1446, doi: 10.1038/eye.2016.143, indexed in Pubmed: 27419840.

13. Donnenfeld ED, Comstock TL, Proksch JW. Human aqueous humor concentrations of besifloxacin, moxifloxacin, and gatifloxacin after topical ocular application. J Cataract Refract Surg. 2011; 37(6): 1082-1089, doi: 10.1016/j.jcrs.2010.12.046, indexed in Pubmed: 21596251.

14. Kıvanç SA, Kıvanç M, Bayramlar H. Microbiology of corneal wounds after cataract surgery: biofilm formation and antibiotic resistance patterns. J Wound Care. 2016; 25(1): 12, 14-9, doi: 10.12968/ jowc.2016.25.1.12, indexed in Pubmed: 26762493. 